\title{
Associação entre a violência comunitária e no local de trabalho e a qualidade do sono de profissionais da saúde: estudo transversal
}

\author{
Association between community-based and workplace violence \\ and the sleep quality of health professionals: a cross-sectional study
}

Fabrícia Bezerra de Castro Alves Silveira (https://orcid.org/0000-0003-1728-8007) ${ }^{1}$

José Claudio Garcia Lira Neto (https://orcid.org/0000-0003-2777-1406) ${ }^{2}$

Carleara Weiss (https://orcid.org/0000-0003-4421-0985) ${ }^{3}$

Márcio Flávio Moura de Araújo (https://orcid.org/0000-0001-8832-8323) ${ }^{4}$

\footnotetext{
${ }^{1}$ Prefeitura Municipal de Fortaleza. R. São José

01, Centro. 60765-165 Fortaleza CE Brasil. fabricia-alves@uol.com.br ${ }^{2}$ Departamento de Enfermagem, Universidade Federal do Piauí. Floriano PI Brasil.

${ }^{3}$ Departamento de

Farmacologia e Toxicologia, Faculdade de Medicina e Ciências Biomédicas, Universidade do Estado de Nova York. Albany NY EUA.

${ }^{4}$ Departamento de Saúde da Família, Fundação Oswaldo Cruz. Eusébio CE Brasil.
}

\begin{abstract}
Community-based and workplace violence against health professionals has caused feelings of vulnerability, emotional exhaustion, and psychological changes, reflecting on sleep quality disorders. Thus, we aimed to analyze the association between community and workplace violence and the sleep quality of health professionals working in primary health care. The study was conducted with 286 health professionals who worked in PHC in one of the largest cities in Brazil. The primary outcome was sleep quality as assessed by the Pittsburgh Sleep Quality Index. Most of the participants $(69.2 \% / p=0.074)$ were classified as "bad sleepers", and nurses (73.3\%/ $p=0.049$ ) showed the worst sleep quality. Additionally, most professionals suffered some type of violence at work $(p<0.001)$, which impaired professional activities $(p=0.010)$, forcing them to change their behavior $(p<0.001)$ and consider changing the workplace location $(p=0.051)$. The results showed that professionals have been experiencing moments of fear, anxiety, and stress associated with workplace and community violence. This highlights the need for public policies geared to occupational health.
\end{abstract}

Key words Workplace Violence, Sleep, Health Personnel, Primary Health Care
Resumo A violência comunitária e no local de trabalho contra os profissionais de saúde tem causado sentimentos de vulnerabilidade, desgaste emocional e alterações psicológicas, refletindo em distúrbios na qualidade do sono. Assim, buscou-se analisar a associação entre violência comunitária e no local de trabalho e a qualidade do sono de profissionais de saúde que atuam na atenção primária à saúde. O estudo foi realizado com 286 profissionais de saúde que atuavam na atenção primária à saúde, em uma das maiores cidades do Brasil. O desfecho primário foi a qualidade do sono, avaliada pelo Índice de Qualidade do Sono de Pittsburgh. A maioria dos participantes $(69,2 \% / p=0,074)$ foi classificada como "maus dormidores", sendo os enfermeiros (73,3\%/ $p=0,049$ ) os que apresentaram a pior qualidade de sono. Ademais, grande parte dos profissionais sofreram algum tipo de violencia durante o trabalho $(p<0,001)$, que prejudicou as atividades profissionais ( $p=0,010)$, obrigando-os a mudar de conduta $(p<0,001)$, e a cogitar mudança do local de trabalho ( $p=0,051)$. Os resultados mostraram que os profissionais têm apresentado episódios de medo, ansiedade e estresse, relacionados à violência no local de trabalho. Isso evidencia a necessidade de políticas públicas voltadas à saúde ocupacional. Palavras-chave Violência no Trabalho, Sono, Profissionais da Saúde, Atenção Primária à Saúde 


\section{Introdução}

A violência no local de trabalho contra profissionais da saúde tem sido um grande desafio ocupacional, especialmente pelo impacto significativo sobre o bem-estar físico e mental desses trabalhadores, limitando o desempenho na prestação dos serviços e na satisfação laboral. Episódios de violência verbal ou física são comuns em setores hospitalares, como, por exemplo, nas enfermarias psiquiátricas ou nos setores de urgência e emergência, assim como também, em unidades de Atenção Primária à Saúde (APS), o que acaba afetando o funcionamento e a eficiência de todo o sistema de saúde a curto, médio e longo prazo ${ }^{1-3}$.

Experiências de violência no local de trabalho podem estar associadas a prejuízos físicos e a consequências emocionais e psicológicas como o medo, a ansiedade, o estresse pós-traumático, a culpa, ou mesmo, à diminuição na qualidade do sono em diferentes proporções ${ }^{4}$. Uma revisão sistemática e metanálise mostrou que os trabalhadores expostos à violência no local de trabalho manifestam problemas relacionados tanto à quantidade do sono, quanto à qualidade do mesmo, ou seja, dificuldade em adormecer, interrupções frequentes, insônia e pesadelos. Isso contribui para a perda da produtividade, aumento de comportamento hostil e agressivo, erros de conduta e outros desfechos negativos, influenciando na segurança do paciente 4 .

Esse cenário parece se agravar, ainda mais, quando a força de trabalho é majoritariamente feminina, os locais de trabalho estão sediados em ambientes geográficos e sociais marcados por vulnerabilidades, e quando as escalas são noturnas ${ }^{3}$. Ademais, o fenômeno da violência no local de trabalho e na comunidade em que atuam os profissionais da saúde são comumente gerados por parentes ou acompanhantes dos pacientes, contra médicos. Por outro lado, a literatura não é unânime quanto aos achados em destaque, e pesquisadores ponderam que talvez a percepção e atitude dos médicos em relação à violência tenha sido diferente dos enfermeiros - grupo que recebem um alto nível de assédio emocional, físico e sexual ${ }^{1}$.

Dados de revisões prévias apontam lacunas sobre investigações a respeito de associações entre a violência no local de trabalho contra profissionais da saúde, especialmente daqueles da APS $^{5,6}$. Somado a isto, de nosso conhecimento, não há dados publicados sobre o efeito da violência na comunidade e no local de trabalho e a qualidade do sono dos profissionais de saúde no país. Tampouco, é identificável informações sobre a perspectiva desses trabalhadores de saúde sobre a influência da violência comunitária na qualidade do seu sono. A partir disso, hipotetizamos que os profissionais de cuidados primários em saúde que trabalham em regiões violentas podem ser mais vulneráveis à má qualidade do sono. Uma hipótese secundária que aventamos é de que profissionais considerados "maus dormidores" podem relatar perspectivas negativas sobre o efeito da violência comunitária e do local de trabalho em seu sono. Uma vez aprofundadas essas questões, faz-se possível traçar estratégias de mitigação e prevenção desse risco ocupacional, na tentativa de melhorar a qualidade de vida dos profissionais de saúde.

Desse modo, o objetivo primário desse trabalho foi analisar a associação entre violência comunitária e na região do local de trabalho, representada pela taxa de homicídios, e a qualidade do sono entre profissionais da APS de Fortaleza, Brasil. O objetivo secundário foi analisar as perspectivas dos profissionais de saúde da APS sobre a violência no local de trabalho e o seu sono.

\section{Método}

\section{Delineamento do estudo}

Trata-se de um estudo transversal, realizado em seis regiões de saúde, localizadas na cidade de Fortaleza, na região nordeste do Brasil, entre os meses de julho e novembro de 2019. O estudo foi aprovado pelo Comitê de Ética em Pesquisa com Seres Humanos do Instituto Pitágoras.

\section{População e amostra}

A população alvo abrangeu os profissionais da saúde de nível superior (médicos, enfermeiros e cirurgiões-dentistas), que trabalhavam em equipes da Estratégia Saúde da Família (ESF), na cidade de Fortaleza. Atualmente o número de profissionais de nível superior devidamente cadastrados nas equipes da ESF é de 1.105, sendo 355 médicos, 463 enfermeiros e 286 cirurgiõesdentistas, distribuídos nas seis regiões da cidade.

Para o cálculo da amostra, estabelecemos como critério de inclusão: ser médico, enfermeiro ou cirurgião-dentista atuante há, pelo menos, um mês ininterrupto na mesma ESF, independente de sexo e idade. Por outro lado, excluímos da amostra, os profissionais em uso de psicotrópicos há mais de 12 meses, ou aqueles com relato 
de diagnóstico para problema de cunho neurológico e/ou psiquiátrico que interferisse no sono.

De posse disso, realizamos cálculo amostral com base em fórmula para população finita.

$$
\mathrm{n}=\frac{N \cdot Z^{2} \cdot p \cdot(1-p)}{Z^{2} \cdot p \cdot(1-p)+e^{2} \cdot(N-1)}
$$

Onde: $\mathrm{n}=$ amostra calculada; $\mathrm{N}=$ população (1.105); Z=variável normal padronizada associada ao nível de confiança $(1,96) ; \mathrm{p}=$ Verdadeira probabilidade do evento $(0,5)$ (devido o desconhecimento de estudos prévios na temática qualidade do sono de profissionais da APS); E=Erro amostral (5\%).

Utilizamos como desfecho a prevalência de má qualidade do sono, pois desconhecemos estudos que tenham associado má qualidade do sono de profissional da APS com a violência. Após os cálculos, a amostra final foi composta por 286 participantes. Este valor foi estratificado em seis estratos (em face das seis regiões de Fortaleza), conforme o percentual representativo de cada categoria profissional de saúde, por região, em relação ao total dos profissionais de saúde de Fortaleza (100\%), Brasil, como disposto no Quadro 1.

\section{Variáveis preditoras do estudo}

As variáveis abordadas neste bloco foram agrupadas em informações sociodemográficas e sobre violência. No caso das informações sociodemográficas, indagamos itens como idade (em anos), sexo, categoria profissional (médico, cirurgião-dentista ou enfermeiro), e tempo de serviço na unidade de cuidados primários (em meses). Ainda questionamos nome do serviço, bairro e a região de localização da unidade de cuidados primários.
Investigamos a violência comunitária através da taxa de homicídios por 100 mil habitantes nas seis regiões de Fortaleza, no ano de 2018, já que essa taxa deriva da taxa de mortalidade por causas externas, resultantes de violência.

Para o cálculo da taxa de mortalidade foi necessário acessar os dados sobre mortalidade, através de sistema TABNET (sistema digital de acesso livre, disponível em http://intranet.sms.fortaleza. ce.gov.br), para identificar os óbitos por causas externas específicos por homicídios (óbitos por agressão - códigos X85 a Y09 do capítulo XX Causas externas de morbidade e mortalidade, da 10a Revisão da Classificação Internacional de Doenças [CID-10]) por região.

O cálculo da taxa de mortalidade por homicídios por 100 mil habitantes foi realizado através do número de óbitos de residentes por homicídios (agressões) divididos pela população total residente por região, seguido de multiplicação por 100 mil. Dessa forma, obtivemos a mensuração da violência por região no ano de 2018 como exposto no Quadro 2.

\section{Desfecho}

A variável desfecho do nosso estudo foi a qualidade do sono, segundo o Índice de Qualidade do Sono de Pittsburgh (IQSP). Os profissionais foram classificados em bons ou maus dormidores.

\section{Coleta de dados e Instrumentos}

A equipe de coleta dos dados visitou 71 Unidades Básicas de Saúde para entrevistar os profissionais de saúde, durante o segundo semestre do ano de 2019. Durante as visitas, os profissionais

Quadro 1. Estratificação da amostra de acordo com a categoria profissional e região de Fortaleza, Ceará, Brasil, 2019.

\begin{tabular}{|l|c|c|c|c|c|c|c|}
\hline Região & $\begin{array}{c}\text { Número de } \\
\text { médicos }\end{array}$ & $\begin{array}{c}\text { Amostra de } \\
\text { médicos }\end{array}$ & $\begin{array}{c}\text { Número de } \\
\text { Enfermeiros }\end{array}$ & $\begin{array}{c}\text { Amostra de } \\
\text { Enfermeiros }\end{array}$ & $\begin{array}{c}\text { Número de } \\
\text { cirurgiões- } \\
\text { dentistas }\end{array}$ & $\begin{array}{c}\text { Amostra de } \\
\text { cirurgiões- } \\
\text { dentistas }\end{array}$ & $\begin{array}{c}\text { Total da } \\
\text { amostra }\end{array}$ \\
\hline 1 & 55 & 14 & 68 & 18 & 34 & 9 & 41 \\
\hline 2 & 44 & 11 & 47 & 12 & 43 & 11 & 34 \\
\hline 3 & 60 & 16 & 78 & 20 & 43 & 11 & 47 \\
\hline 4 & 36 & 9 & 48 & 13 & 35 & 9 & 31 \\
\hline 5 & 65 & 17 & 97 & 25 & 46 & 12 & 54 \\
\hline 6 & 95 & 25 & 125 & 32 & 86 & 22 & 79 \\
\hline Total & 355 & 92 & 463 & 120 & 287 & 74 & 286 \\
\hline
\end{tabular}

Fonte: Elaborado pelos autores. 
Quadro 2. Taxa de mortalidade por homicídio por 100 mil habitantes, na região de Fortaleza, Ceará, Brasil, 2018.

\begin{tabular}{|c|c|}
\hline Região & $\begin{array}{c}\text { Taxa de mortalidade por homicídio } \\
\text { por } \mathbf{1 0 0} \text { mil habitantes }\end{array}$ \\
\hline 1 & 55,32 \\
\hline 2 & 40,59 \\
\hline 3 & 51,72 \\
\hline 4 & 43,48 \\
\hline 5 & 91,13 \\
\hline 6 & 61,98 \\
\hline Fortaleza & 60,15 \\
\hline
\end{tabular}

Fonte: Elaborado pelos autores.

de saúde atuantes foram abordados e questionados sobre o interesse em participar da pesquisa. Após a aplicação dos critérios de elegibilidade e da assinatura do Termo de Consentimento Livre e Esclarecido, deu-se início à coleta dos dados.

Para tanto, foi elaborado um instrumento contendo 28 questões, divididas em três partes, a saber: características sociodemográficas (sexo, idade, região onde se localiza a unidade de saúde, tempo de trabalho), avaliação do sono segundo o Pittsburgh Sleep Quality Index (PSQI), adaptada e validada em português do Brasil sob o nome de Índice de Qualidade do Sono de Pittsburgh $(\mathrm{IQSP})^{7}$. Por sua vez, as informações sobre a violência foram avaliadas por meio de questões objetivas sobre a perspectiva do entrevistado acerca do seu sono e a violência comunitária e no seu local de trabalho. A exemplo disso, as seguintes perguntas foram feitas: Você considera o território de atuação violento? A violência territorial prejudicou atividades profissionais? A violência territorial obrigou você a mudar sua conduta? Você foi vítima de violência no serviço ou comunidade? entre outras. Os profissionais foram abordados em três turnos diferentes (manhã, tarde e noite), em sala reservada para tal, garantindo a privacidade das informações.

\section{Análise dos dados}

Os dados colhidos foram digitados e tabulados no software livre Epi Data versão 3.1 e na sequência analisados por meio do software SPSS versão 23. Para as variáveis qualitativas foram calculadas frequência absoluta e relativa. As variáveis quantitativas foram resumidas por meio das estatísticas: média, desvio padrão, quartis, mínimo e máximo. A normalidade das variáveis quantitativas foi verificada por meio do teste de Shapiro-Wilk. A associação entre variáveis qualitativas foi verificada por meio dos testes Quiquadrado e exato de Fisher e a comparação de grupos entre variáveis quantitativas foi verificada por meio dos testes de Mann-Whitney.

Após identificar as características que apresentaram relação significante com a qualidade do sono, um modelo de regressão de Poisson foi ajustado para evitar possíveis variáveis confundidoras. A magnitude da associação entre as variáveis foi expressa por meio de estimativas pontuais e intervalares de razões de prevalências.

A regressão de Poisson foi utilizada para estimar a razão de prevalência ajustada, ao invés da razão de chances como é o caso da regressão logística.

Os resultados foram apresentados em gráficos e tabelas. Para todos os procedimentos inferenciais foi adotado um nível de significância de $5 \%$.

\section{Resultados}

\section{Qualidade do sono entre os profissionais de saúde da Atenção Primária à Saúde}

Nesse estudo, a maioria dos participantes eram mulheres $(73,8 \%)$, com idade entre $40 \mathrm{e}$ 49 anos (40,6\%), e enfermeiras (42\%). A partir disso, na avaliação do sono dos profissionais de saúde, notou-se que aproximadamente metade da amostra relatou boa qualidade subjetiva do sono $(52,7 \%)$, e duração do sono entre 6-7 horas $(51,4 \%)$. Quanto ao componente latência do sono, as opções de resposta 16-30 minutos $(30,8 \%)$ e $31-61$ minutos (35\%) foram predominantes, segundo o IQSP. No componente eficiência do sono, preponderou a resposta com a opção $\geq 85 \%$ (76,2\%).

A maioria dos participantes foi classificado como "maus dormidores" (65,7\%). Os profissionais de saúde em cuidados primários com maior prevalência de má qualidade do sono foram os enfermeiros $(73 \% / \mathrm{p}=0,049)$, segundo o IQSP. Não identificamos associação estatisticamente significante entre má qualidade do sono e as variáveis sexo $(\mathrm{p}=0,074)$, faixa etária $(\mathrm{p}=0,989)$, região do local de trabalho $(\mathrm{p}=0,518)$ e tempo de trabalho na unidade de cuidados primários $(\mathrm{p}=0,957)$, conforme mostra a Tabela 1 .

Os participantes considerados "maus dormidores", conforme o IQSP, apresentaram elevadas 
Tabela 1. Distribuição das características sociodemográficas, de acordo com a classificação da qualidade do sono. Fortaleza, Ceará, Brasil, 2019 ( $\mathrm{n}=286)$.

\begin{tabular}{|c|c|c|c|c|c|c|}
\hline \multirow{2}{*}{ Variáveis } & \multicolumn{2}{|c|}{ Mau dormidor $(n=188)$} & \multicolumn{2}{|c|}{ Bom dormidor $(n=98)$} & \multirow{2}{*}{ RP (IC95\%) } & \multirow{2}{*}{ p-valor } \\
\hline & $\mathbf{n}$ & $\%$ & $\mathbf{n}$ & $\%$ & & \\
\hline Sexo & & & & & & $0,074^{1}$ \\
\hline Masculino & 43 & 57,3 & 32 & 42,7 & 1 & \\
\hline Feminino & 145 & 68,7 & 66 & 31,3 & $1,2(0,97-1,49)$ & \\
\hline Faixa etária & & & & & & $0,989^{2}$ \\
\hline 23 a 29 & 28 & 68,3 & 13 & 31,7 & $1,07(0,74-1,53)$ & \\
\hline 30 a 39 & 64 & 66,7 & 32 & 33,3 & $1,04(0,75-1,44)$ & \\
\hline 40 a 49 & 75 & 64,7 & 41 & 35,3 & $1,01(0,73-1,40)$ & \\
\hline 50 a 59 & 16 & 64,0 & 9 & 36,0 & 1 & \\
\hline$\geq 60$ & 5 & 71,4 & 2 & 28,6 & $1,12(0,64-1,94)$ & \\
\hline Categoria profissional & & & & & & $0,049^{1}$ \\
\hline Médico & 58 & 63,0 & 34 & 37,0 & $1,11(0,86-1,43)$ & \\
\hline Enfermeiro & 88 & 73,3 & 32 & 26,7 & $1,29(1,03-1,62)$ & \\
\hline Cirurgião-dentista & 42 & 56,8 & 32 & 43,2 & 1 & \\
\hline $\begin{array}{l}\text { Região da cidade em que } \\
\text { se localiza a unidade de } \\
\text { cuidados primários em saúde }\end{array}$ & & & & & & $0,518^{1}$ \\
\hline Região 1 & 26 & 63,4 & 15 & 36,6 & $1,10(0,80-1,53)$ & \\
\hline Região 2 & 24 & 70,6 & 10 & 29,4 & $1,23(0,90-1,69)$ & \\
\hline Região 3 & 34 & 72,3 & 13 & 27,7 & $1,26(0,94-1,68)$ & \\
\hline Região 4 & 23 & 74,2 & 8 & 25,8 & $1,29(0,95-1,76)$ & \\
\hline Região 5 & 31 & 57,4 & 23 & 42,6 & 1 & \\
\hline Região 6 & 50 & 63,3 & 29 & 36,7 & $1,1(0,83-1,47)$ & \\
\hline $\begin{array}{l}\text { Tempo de trabalho na } \\
\text { unidade de cuidados } \\
\text { primários em saúde }\end{array}$ & 72,0 & $156,0)$ & 72,0 & $44,0)$ & - & $0,957^{3}$ \\
\hline
\end{tabular}

taxas de qualidade subjetiva do sono muito ruim e ruim $(p<0,001)$, latência do sono $>60$ minutos $(\mathrm{p}<0,001)$, duração do sono $<5$ horas/dia ( $<<0,001)$, eficiência habitual do sono $<65 \%$ e entre $65-74 \%$.

Observamos nos participantes classificados como "maus dormidores" que as variáveis distúrbios do sono $\geq 3$ vezes na semana $(p<0,001)$, uso de drogas para dormir $\geq 3$ vezes na semana $(\mathrm{p}<0,001)$ e sonolência diurna $\geq 3$ vezes na semana $(\mathrm{p}<0,001)$, apresentaram diferenças estatisticamente significantes em relação aos bons dormidores. A propósito, durante o cálculo do IQSP, foi justamente no domínio distúrbios do sono que averiguamos maior pontuação e menor desvio padrão ( $1,4 \pm 0,6$ pontos).

\section{Perspectivas dos profissionais de cuidados primários acerca da violência na região do local de trabalho e o seu sono}

A maioria dos entrevistados $(60,8 \%)$ considera a região onde trabalham muito violenta. Fato é que $80,1 \%$ dos participantes relataram ter sofrido algum episódio de violência dentro do serviço de saúde, ou seja, em seus respectivos locais de trabalho, entre as seis regiões que compõe a cidade de Fortaleza. Neste caso, as frequências de situações de violência mais relatadas foram $1-2$ vezes $(39,2 \%)$ e $\geq 3$ vezes $(40,9 \%)$. Neste último caso, duas características estiveram associadas estatisticamente a essa resposta, a saber: ser enfermeiro $(50 \%, \mathrm{p}=0,011)$ ou mau dormidor $(71,9 \%, \mathrm{p}=0,001)$.

Observamos um resultado oposto quando investigamos relatos de violência nas atividades de trabalho realizadas fora do prédio da UBS, sen- 
do que uma parcela expressiva dos entrevistados $(64,7 \%)$ relatou nunca ter sofrido algum tipo de violência.

Do ponto de vista da maioria dos entrevistados $(89,1 \%)$, a violência no local de trabalho prejudicou o desenvolvimento das suas atividades profissionais, em mais de uma ocasião, sendo $37,1 \%$ de $1-2$ vezes, e $44,8 \% \geq 3$ vezes. Esta última opção de resposta foi dada principalmente pelos participantes considerados maus dormidores ( $\mathrm{p}=0,001)$, conforme mostra a Tabela 2 .

Outros relatos predominantes ( $\geq 3$ vezes), devido a violência comunitária e do local de trabalho, entre os maus dormidores foram o sentimento de medo, ansiedade ou estresse $(\mathrm{p}=0,011)$, episódios de demora para adormecer $(\mathrm{p}=0,001) \mathrm{e}$ a ocorrência de pesadelos $(\mathrm{p}=0,001)$ (Tabela 2$)$.

A prevalência de "maus dormidores" entre os profissionais de saúde sobressaiu-se em todas as regiões da cidade analisada. Entretanto, com base no teste de Mann-Whitney, não observamos associação estatisticamente significante entre taxa de homicídios e a qualidade do sono dos profissionais entrevistados $(\mathrm{p}=0,191)$.

Ao associarmos a variável má qualidade do sono dos profissionais com as taxas de homicídios (por regional e bairros da cidade de Fortaleza, Brasil), não foram evidenciadas diferenças estatisticamente significantes entre médicos $(\mathrm{p}=0,191)$, enfermeiros $(\mathrm{p}=0,069)$ e cirurgiões-dentistas $(\mathrm{p}=0,467)$. Ademais, a taxa de homicídio não influenciou no relato de demora a dormir devido pensar na violência sofrida $(\mathrm{p}=0,997)$. Contudo, na região 2 , o relato de pesadelos predominou naqueles que viviam em locais cuja mediana de taxa de violência foi maior $(\mathrm{p}=0,015)$.

Após o ajuste, as variáveis mudança de conduta e demora a dormir ao pensar na violência comunitária e do local de trabalho permaneceram significantes no modelo. Os profissionais que relataram mudança de conduta $\geq 3$ vezes apresentaram prevalência de má qualidade do sono $42 \%$ maior em relação àqueles que não relataram mudança de conduta. Por sua vez, os participantes que demoram a dormir, apresentaram prevalência de má qualidade do sono $55 \%$ maior (Tabela 3).

\section{Discussão}

Em nosso estudo, $65,7 \%$ dos profissionais de saúde atuantes na APS foram considerados "mau dormidores”, segundo o IQSP. Desses, 73\% eram enfermeiros $(\mathrm{p}=0,049)$. Sobre isso, a literatura tem traçado um paralelo entre as longas jornadas de trabalho e escalas noturnas e o comprometimento do sono (IQSP > 7) de profissionais da enfermagem, o que pode estar diretamente relacionado à diminuição da qualidade da assistência prestada, bem como, ao comprometimento do ciclo sono-vigília, na qualidade de vida e no bem -estar físico, psíquico e social desses trabalhadores $^{8-12}$. Evidências de uma metanálise conduzida com 53 estudos observacionais concluíram que os enfermeiros são os profissionais mais suscetíveis a má qualidade do sono ${ }^{13}$.

No Brasil, os enfermeiros da APS são os profissionais mais envolvidos na gestão organizacional e na mediação do trabalho das demais categorias profissionais. Ademais, esses profissionais são encarregados de muitas outras atividades que extrapolam os muros das unidades de saúde, o que pode aumentar o escopo de preditores responsáveis pelo comprometimento da qualidade do sono.

Ainda no contexto da APS, dados de um estudo nacional apontaram que a insônia é um problema recorrente citado por profissionais de saú$\mathrm{de}^{14,15}$. Além disso, a insatisfação com a qualidade do sono e a dificuldade para dormir também são frequentemente registrados como sequelas causadas pelo ambiente laboral da APS ${ }^{16,17}$. Provavelmente, isto explique o fato de uma parcela substancial dos participantes desta pesquisa revelar usar medicamentos para dormir. Este achado é similar ao de outros estudos prévios consultados, cuja variação de má qualidade do sono oscilou entre $17,7 \%$ e $85 \%$, e que colocam o enfermeiro como um dos principais usuários desse tipo de medicamento $0^{9,10,15}$. Acreditamos que nas unidades de cuidados primários no Brasil há fatores que cooperam para isso, como a complexidade das demandas das populações atendidas, a pouca governabilidade dos profissionais de saúde na resolução de problemas estruturais e de processos de trabalho, e a violência no local de trabalho.

De fato, a violência no ambiente laboral pode ser considerada um fator estressante e que gera repercussões negativas à vida das pessoas envolvidas. Quando em regiões marcadas pela incivilidade e vulnerabilidade social, o risco ocupacional e os distúrbios no sono tornam-se ainda mais presentes.

Em nosso estudo, 60,8\% dos entrevistados consideraram a região em que trabalhavam como violenta, e $80,1 \%$ da amostra relatou ter sofrido algum tipo de violência durante a atividade profissional. Uma revisão sistemática e 
Tabela 2. Distribuição da percepção dos profissionais de saúde quanto à violência pela qualidade do sono. Fortaleza, Ceará, Brasil, 2019 (n=286).

\begin{tabular}{|c|c|c|c|c|c|c|}
\hline \multirow[t]{2}{*}{ Variáveis } & \multicolumn{2}{|c|}{$\begin{array}{c}\text { Mau dormidor } \\
(\mathbf{n}=\mathbf{1 8 8})\end{array}$} & \multicolumn{2}{|c|}{$\begin{array}{c}\text { Bom dormidor } \\
(\mathrm{n}=98)\end{array}$} & \multirow[t]{2}{*}{ RP (IC95\%) } & \multirow[t]{2}{*}{ p-valor } \\
\hline & $\mathbf{n}$ & $\%$ & $\mathbf{n}$ & $\%$ & & \\
\hline Considera o território de atuação & & & & & & $0,157^{2}$ \\
\hline Não considero uma área violenta & 3 & 42,9 & 4 & 57,1 & 1 & \\
\hline Considero uma área pouca violenta & 64 & 61,0 & 41 & 39,0 & $1,42(0,6-3,39)$ & \\
\hline Considero uma área muito violenta & 121 & 69,5 & 53 & 30,5 & $1,62(0,69-3,84)$ & \\
\hline Violência territorial prejudicou atividades profissionais & & & & & & $0,010^{1}$ \\
\hline Nenhuma vez & 24 & 48,0 & 26 & 52,0 & 1 & \\
\hline 1 ou 2 vezes & 71 & 67,0 & 35 & 33,0 & $1,4(1,02-1,92)$ & \\
\hline$\geq 3$ vezes & 92 & 71,9 & 36 & 28,1 & $1,5(1,1-2,04)$ & \\
\hline Violência territorial obrigou a mudar sua conduta & & & & & & $<0,001^{1}$ \\
\hline Nenhuma vez & 36 & 43,9 & 46 & 56,1 & 1 & \\
\hline 1 ou 2 vezes & 72 & 69,9 & 31 & 30,1 & $1,59(1,21-2,1)$ & \\
\hline$\geq 3$ vezes & 80 & 79,2 & 21 & 20,8 & $1,8(1,39-2,35)$ & \\
\hline $\begin{array}{l}\text { Foi vítima de violência durante atividades laborais } \\
\text { dentro do serviço }\end{array}$ & & & & & & $<0,001^{1}$ \\
\hline Nenhuma vez & 25 & 44,6 & 31 & 55,4 & 1 & \\
\hline 1 ou 2 vezes & 70 & 62,5 & 42 & 37,5 & $1,4(1,01-1,94)$ & \\
\hline$\geq 3$ vezes & 92 & 78,6 & 25 & 21,4 & $1,76(1,3-2,39)$ & \\
\hline $\begin{array}{l}\text { Foi vítima de violência durante atividades laborais } \\
\text { dentro da comunidade }\end{array}$ & & & & & & $0,007^{1}$ \\
\hline Nenhuma vez & 110 & 59,5 & 75 & 40,5 & 1 & \\
\hline 1 ou 2 vezes & 45 & 72,6 & 17 & 27,4 & $1,22(1,01-1,48)$ & \\
\hline$\geq 3$ vezes & 31 & 83,8 & 6 & 16,2 & $1,41(1,17-1,7)$ & \\
\hline $\begin{array}{l}\text { Sentiu medo, ansiedade ou estresse devido violência no } \\
\text { território }\end{array}$ & & & & & & $0,001^{2}$ \\
\hline Nenhuma vez & 22 & 45,8 & 26 & 54,2 & 1 & \\
\hline Não considero o território violento & 1 & 100,0 & 0 & 0,0 & $2,18(1,6-2,97)$ & \\
\hline 1 ou 2 vezes & 71 & 62,8 & 42 & 37,2 & $1,37(0,98-1,92)$ & \\
\hline$\geq 3$ vezes & 93 & 76,2 & 29 & 23,8 & $1,66(1,2-2,3)$ & \\
\hline Demorou a dormir ao pensar na violência territorial & & & & & & $<0,001^{1}$ \\
\hline Nenhuma vez & 78 & 51,3 & 74 & 48,7 & 1 & \\
\hline 1 ou 2 vezes & 67 & 78,8 & 18 & 21,2 & $1,54(1,27-1,86)$ & \\
\hline$\geq 3$ vezes & 43 & 89,6 & 5 & 10,4 & $1,75(1,45-2,1)$ & \\
\hline $\begin{array}{l}\text { Teve pesadelos relacionado a violência no serviço/ } \\
\text { território }\end{array}$ & & & & & & $<0,001^{1}$ \\
\hline Nenhuma vez & 125 & 59,2 & 86 & 40,8 & 1 & \\
\hline 1 ou 2 vezes & 42 & 85,7 & 7 & 14,3 & $1,45(1,23-1,7)$ & \\
\hline$\geq 3$ vezes & 21 & 80,8 & 5 & 19,2 & $1,36(1,1-1,7)$ & \\
\hline $\begin{array}{l}\text { Acordou desmotivado para ir ao trabalho devido a } \\
\text { violência no serviço/território }\end{array}$ & & & & & & $<0,001^{1}$ \\
\hline Nenhuma vez & 62 & 52,5 & 56 & 47,5 & 1 & \\
\hline 1 ou 2 vezes & 57 & 72,2 & 22 & 27,8 & $1,37(1,1-1,71)$ & \\
\hline$\geq 3$ vezes & 69 & 78,4 & 19 & 21,6 & $1,49(1,22-1,83)$ & \\
\hline $\begin{array}{l}\text { Cogitou mudar o local de trabalho devido a violência no } \\
\text { serviço/território }\end{array}$ & & & & & & $0,051^{1}$ \\
\hline Nenhuma vez & 106 & 63,5 & 61 & 36,5 & 1 & \\
\hline 1 ou 2 vezes & 39 & 60,0 & 26 & 40,0 & $0,95(0,75-1,19)$ & \\
\hline$\geq 3$ vezes & 43 & 79,6 & 11 & 20,4 & $1,25(1,05-1,5)$ & \\
\hline
\end{tabular}

${ }^{1}$ Teste qui-quadrado; ${ }^{2}$ Teste exato de Fisher.

Fonte: Elaborado pelos autores. 
Tabela 3. Modelo de regressão de Poisson para a variável má qualidade do sono dos profissionais.

\begin{tabular}{lrc}
\hline \multicolumn{1}{c}{ Variáveis } & RP (IC95\%) & p-valor \\
\hline $\begin{array}{l}\text { A violência na } \\
\text { comunidade obrigou }\end{array}$ & & \\
os profissionais a & & \\
mudarem de conduta? & & \\
$\quad$ Nenhuma vez & 1 & \\
$\quad 1$ ou 2 vezes & $1,42(1,07-1,89)$ & 0,014 \\
$\quad$ 3 vezes & $1,42(1,06-1,89)$ & 0,019 \\
$\begin{array}{l}\text { Profissional demorou } \\
\text { muito para dormir ao }\end{array}$ & & \\
pensar sobre violência & & \\
na comunidade? & & \\
$\quad$ Nenhuma vez & & \\
$\quad 1$ ou 2 vezes & $1,38(1,13-1,69)$ & 0,002 \\
$\geq 3$ vezes & $1,55(1,25-1,91)$ & $<0,001$ \\
\hline
\end{tabular}

Fonte: Elaborado pelos autores.

metanálise que buscou avaliar a prevalência de violência contra enfermeiras iranianas mostrou que a violência verbal, física, sexista e racista no local de trabalho e a ameaça contra essas profissionais foi de 80,8\% (IC95\% [74,2-86,0]), 24,8\% (IC95\% [17,4-34]), 6,7\% (IC95\% [4-9,2]), 14,6\% (IC95\% [10,1-20,7]) e 44\% (IC95\% [30,1-58,8]), respectivamente ${ }^{18}$, resultando em uma experiência marcada por sensações de medo, ansiedade, estresse, demora para adormecer e pesadelos constantes.

A respeito disso, dos 188 profissionais considerados maus dormidores, $80,5 \%$ referiram ter pesadelos, $89,6 \%$ afirmaram demora para adormecer, e $76,2 \%$ expressaram ter sentido medo, ansiedade e estresse quando experienciaram episódios de violência no local de trabalho e na comunidade, o que acaba prejudicando o desempenho do exercício profissional - fato confirmado por quase $90 \%$ dos entrevistados.

Ressalta-se que, a maioria dos profissionais classificados como maus dormidores relataram sofrer violência dentro dos serviços de cuidados primários e não na comunidade em si. Este achado diverge de artigo publicado há cerca de 15 anos, no Brasil, que constatou que atividades como as visitas domiciliares são um fator de risco laboral, à medida que a sensação de segurança da estrutura física da unidade de saúde primária é rompida ${ }^{19}$. Apesar da variável qualidade do sono não ter sido empregada neste estudo ora citado, acreditamos que a divergência seja justificável pela questão cronológica, pois hoje, o país é mais violento em muitos âmbitos sociais, inclusive dentro dos serviços de saúde.

Apesar disso, muitas das análises realizadas acerca das variáveis de violência e sono dos profissionais não foram estatisticamente significantes. É possível que isso derive da visão do profissional sobre a violência, isto é, a violência é um fenômeno interpretado conforme a época, local e circunstâncias em que ocorre; por isso, há violências toleradas ou condenadas pelos profissionais. Portanto, qualquer análise sobre a temática violência precisa ponderar a subjetividade do relato dos participantes ${ }^{19}$.

Desse modo, é importante que as políticas públicas voltadas à saúde do trabalhador destaquem a importância de se discutir e promover a qualidade do sono dos trabalhadores de saúde da APS, em face das repercussões desse assunto sobre a produtividade e a qualidade de vida.

Esta pesquisa conta com algumas limitações que devem balizar sua análise e interpretação. Adotamos a variável taxa de mortalidade por homicídios como representação de desfecho para violência comunitária, a despeito do caráter multifatorial da violência. Ademais, as taxas de mortalidade por homicídio são referentes ao ano de 2018 e o IQSP avalia o sono nos últimos 30 dias, portanto, não há uma sintonia cronológica entre variável dependente e independente.

Outro aspecto é o fato de que dentro de uma mesma região podem existir bairros, com realidades sociais distintas e com taxas de mortalidade por homicídios bastante diferentes entre eles. Uma possibilidade seria ajustar as análises de acordo com o índice de desenvolvimento humano por bairro, mas não os dados registrados sobre essas informações. Por outro lado, nossa amostra foi representativa e os óbitos são desfechos bem documentados e pouco sujeitos a subnotificação no Departamento de Tecnologia da Informação do Sistema Único de Saúde - SUS (DATASUS).

Desse modo, a nossa sugestão é que outras pesquisas possam ser realizadas com delineamento longitudinal e incorporando ao modelo analítico outras variáveis preditoras e/ou de confusão acerca da relação entre a violência comunitária e a qualidade do sono, não apenas com trabalhadores da saúde, mas também na indústria, comércio, educação e demais setores envolvidos.

\section{Conclusão}

Não observamos associação estatisticamente significativa entre a taxa de homicídios e a qua- 
lidade do sono dos profissionais entrevistados. No entanto, devido à violência comunitária, as pessoas que dormiam mal relataram mais sentimentos de medo, ansiedade ou estresse, episódios de demora para adormecer e a ocorrência de pesadelos. Já aqueles que relataram demora para dormir, devido à violência comunitária, tiveram uma prevalência $55 \%$ maior de má qualidade do sono, em comparação com profissionais que não sofreram qualquer tipo de violência.

\section{Colaboradores}

FBCA Silveira e MFM Araújo contribuíram com a concepção e o delineamento, análise e interpretação dos dados. FBCA Silveira, MFM Araújo, JCG Lira Neto e C Weiss realizaram a redação do artigo e sua revisão crítica. JCG Lira Neto, C Weiss e MFM Araújo contribuíram com a aprovação da versão a ser publicada. 


\section{Referências}

1. Kumari A, Kaur T, Ranjan P, Chopra S, Sarkar S, Baitha U. Workplace violence against doctors: characteristics, risk factors, and mitigation strategies. $J$ Postgrad Med 2020; 66(3):149-154.

2. d'Ettorre G, Mazzotta M, Pellicani V, Vullo A. Preventing and managing workplace violence against healthcare workers in Emergency Departments. Acta Biomed 2018; 89(Supl. 4):28-36.

3. Jatic Z, Erkocevic H, Trifunovic N, Tatarevic E, Keco A, Sporisevic L, Hasanovic E. Frequency and forms of workplace violence in primary health care. Med Arch 2019; 73(1):6-10.

4. Magnavitta N, Di Stasio E, Capitanelli I, Lops EA, Chirico F, Garbarino S. Sleep problems and workplace violence: a systematic review and meta-analysis. Front Neurosci 2019; 13:997.

5. Hall BJ, Xiong P, Chang K, Yin M, Sui XR. Prevalence of medical workplace violence and the shortage of secondary and tertiary interventions among healthcare workers in China. J Epidemiol Community Health. 2018; 72(6):516 518.

6. Crawford CL, Chu F, Judson LH, Cuenca E, Jadalla AA, Tze-Polo L, Kawar LN, Runnels C, Garvida RJ. An integrative review of nurse-to-nurse incivility, hostility, and workplace violence: a GPS for nurse leaders. Nurs Adm Q 2019; 43(2):138-156.

7. Bertolazi AN, Fagondes SC, Hoof L, Dartora EG, Miozzo ICS, Barba MEF, Barreto SSM. Validation of the Brazilian Portuguese version of the Pittsburgh Sleep Quality Index. Sleep Med 2011; 12(1):70-75.

8. Deng X, Liu X, Fang R. Evaluation of the correlation between job stress and sleep quality in community nurses. Medicine (Baltimore) 2020; 99(4):e18822.

9. Rocha MCP, Martino MMF. Stress and sleep quality among registered nurses who use sleeping pills. Acta Paul Enferm 2009; 22(5):658-665.

10. Silva JSX, Silva RM, Cangussu DDD, Moraes-Filho IM, Proença MFR. Qualidade do sono dos profissionais de enfermagem do serviço móvel de atendimento de urgência. REVISA 2019; 8(3):264-272.

11. Palhares VC, Corrente JE, Matsubara BB. Association between sleep quality and quality of life in nursing professionals working rotating shifts. Rev Saude Publica 2014; 48(4):594-601.

12. Carvalho JSV, Oliveira I, Rosa R, Barata C, Fradinho M, Oliveira L, Nogueira F. Sleep quality and quality of life in physicians and nurses working at a Central Hospital. Eur Respir J 2018; 52(62):PA4372.
13. Zeng LN, Yang Y, Wang C, Li X-H, Xiang Y-F, Hall BJ, Ungvari GS, Li CY, Chen C, Chen LG, Cui XL, An FR, Xiang YT. Prevalence of poor sleep quality in nursing staff: a meta-analysis of observational studies. Behav Sleep Med 2020; 18(6):746-759.

14. Camelo SHH, Angerami ELS. Sintomas de estresse nos trabalhadores atuantes em cinco núcleos de saúde da família. Rev Latinoam Enfermagem 2004; 12(1):1421.

15. Martins LF, Laport TJ, Menezes VP, Medeiros PB, Ronzani TM. Esgotamento entre profissionais da Atenção Primária à Saúde. Cien Saude Colet 2014; 19(12):4739-4750.

16. Ferrari GSL, Wadi JML, Ferrari, CKB. Qualidade de vida em profissionais da Estratégia Saúde da Família (ESF) no Vale do Araguaia, Amazônia Legal, Brasil. Scire Salutis 2013; 3(1):78-85.

17. Moura A, Lunardi R, Volpato R, Nascimento V, Bassos T, Lemes A. Fatores associados à ansiedade entre profissionais da Atenção Básica. Rev Port Enf Saude Mental 2018; 19:17-26.

18. Azami M, Moslemirad M, YektaKooshali MH, Rahmati S, Soleymani A, Shamloo MBB, Esmaeilpour-Bandboni M, Khataee M, Khorshidi A, Otaghi M. Workplace violence against Iranian nurses: a systematic review and meta-analysis. Violence Vict 2018; 33(6):1148-1175.

19. Velloso ISC, Araújo MT, Rocha AM, Alves M. A visão dos profissionais de saúde sobre a violência no cotidiano de trabalho em uma Unidade Básica. Rev Min Enf 2005; 9(4):302-305.

Artigo apresentado em 17/07/2020

Aprovado em 24/02/2021

Versão final apresentada em 26/02/2021

Editores-chefes: Romeu Gomes, Antônio Augusto Moura da Silva 